\title{
High-frequency capacitance-voltage characteristics of the heterogeneous structure based on the model of spherical semiconductor particles in a dielectric
}

\author{
A. S. Tonkoshkur and A. V. Ivanchenko* \\ Department of Physics, Electronics and Computer Systems \\ Oles Honchar Dnipropetrovsk National University \\ 72 Gagarin Ave., Dnepropetrovsk 49010, Ukraine \\ *TvanchenkoAV@ukr.net
}

Received 27 March 2016; Revised 10 June 2016; Accepted 6 July 2016; Published 22 August 2016

\begin{abstract}
The dependence of the parameters of the capacitance effect in heterogeneous dispersed two-component structures based on semiconductors from the bulk fraction of the semiconductor component is modeled. The used method for determining the changes of the energy bands bending on the surface of the spherical semiconductor particle by applying dc electric field allowed to calculate the changes of the dipole moment and effective (taking into account the polarization of the free charge) dielectric constant of this semiconductor particle. This result allowed to use the known models of the dielectric constant of two-component structures for the description of the capacitance field effect in the heterogeneous structures. The relations allowing to estimate the value of the bulk donor concentration in the semiconductor component of the matrix of the heterogeneous system and the statistical mixture have been obtained. The approbation of the obtained calculation relations to evaluate the donor concentration in the $\mathrm{ZnO}$ grains of zinc oxide varistor ceramics leads to the correct values that are consistent with estimates of other methods and models. It is established that the sensitivity of the relative dielectric constant to the applied dc electric field is dependent on the bulk fraction of the semiconductor particles in the heterogeneous structures. The bulk fraction of the semiconductor particles significantly affects on the dielectric constant beginning with the values from $\sim 0.8$ for matrix systems and $\sim 0.33$ for statistical mixtures.
\end{abstract}

Keywords: Heterogeneous structure; capacitance-voltage characteristic; dipole moment; donor concentration; varistor ceramics.

\section{Introduction}

The capacitance field effect is known as the method of investigating the physical parameters of the structures for semiconductor devices and heterogeneous materials based on semiconductors, in particular, metal oxide varistor ceramics. ${ }^{1-6}$

The region of the high-frequency capacitance-voltage characteristic is the most acceptable for determining such parameters in the frequency range $\tau_{S}^{-1} \ll \omega \ll \tau_{M}, \tau_{M}$ and $\tau_{S}$ are Maxwell relaxation time and time capture of electrons on localized electronic states. In this region, capacitance of structures decreases with increasing applied dc voltage. ${ }^{6-10}$

This effect is used to determine the height of the intergranular potential barrier $\varphi_{0 S}$ and donor concentration in the bulk of the semiconductor $\mathrm{ZnO}$ crystallites $N_{d}$ of the metal oxide varistor ceramics when studying the influence of impurities (additives), structural defects, which arise during the making and the degradation process. ${ }^{11-15}$ It is known that the method of the capacitance-voltage characteristics is used to evaluate the packing density of varistor ceramics. Also, this method together with other methods (in particular, with the use of measuring current-voltage characteristics) is used to determine the density of surface localized electronic states by energy $N_{S}(E)$ on the boundary and the doping profile of the near-surface zone of $\mathrm{ZnO}$ ceramics crystallites along the normal coordinate $N_{d}(x){ }^{6}$

However, the semi-empirical calculation formulas or expressions are used. They were obtained on the basis of the submission ceramics structure by the one-dimensional model of the counter included Schottky barriers, which correspond to regions of space charge of contacting $\mathrm{ZnO}$ crystallites. ${ }^{16-18}$ Also, in this case the voltage drop on the forward biased Schottky barrier is neglected. ${ }^{6,19,20}$ Estimates $N_{d}$ from the experimental data with using these formulas often give somewhat higher values in relation to the values obtained by other methods. ${ }^{6,20,21}$

The one-dimensional models cannot take into account the following factors: the dispersed character of the structure of the real varistor ceramics, the specifics of the distribution in the structure of the electric fields, the interaction of the electric moments of separate semiconductor particles of the structures, etc. The use of these models to heterogeneous structures with the bulk fraction of semiconductor component $p_{V}$ which varies in a wide range (in particular, to known polymer composites with a wide spectrum of fillers from photoconductor microcrystalline particles ${ }^{22}$ to micro-sized particles of ceramic varistor materials ${ }^{23-25}$ ) is not correct. Also, accounting of the parameter $p_{V}$ is important in

This is an Open Access article published by World Scientific Publishing Company. It is distributed under the terms of the Creative Commons Attribution 4.0 (CC-BY) License. Further distribution of this work is permitted, provided the original work is properly cited. 
connection with the known data on the change of the dielectric constant with increase at applied dc electric voltage in polymer composites with fillers as from the semiconductor and conductive particles, ${ }^{26}$ where the value $p_{V}$ is considerably less.

In this paper, the model of high-frequency capacitancevoltage characteristic of heterogeneous material based on semiconductors is proposed. In this model, heterogeneous material has been presented as the structure in form dispersed two-component medium consisting of spherical inclusions of the semiconductor and ideal insulator. Main attention is paid to the dependence of this capacitance effect from the bulk fraction of the semiconductor component and the analysis of the features manifestation of this effect.

\section{Basic Equations of the Model}

According to the model of the system of spherical semiconductor particles in the dielectric, the equations of the continuity of electric potential and induction which are used to find the dependence of the surface bending of energy bands $Y_{E S}=Y_{E}(a)$ in the spherical semiconductor particle with the radius $a$ from the dc bias voltage $V_{0}$ (at $L / a \ll 1$, where $L$ is the Debye screening length) can be written as

$$
\begin{gathered}
\left.\left(Y_{E}-Y_{0}\right)\right|_{r=a}=\frac{e \psi_{0 d}(a)}{k T}, \\
\left.\varepsilon_{0} \varepsilon_{h}^{\prime} \frac{\partial \psi_{0 d}}{\partial r}\right|_{r=a}-\varepsilon_{0} \varepsilon_{s}^{\prime} \frac{k T}{e}\left(\left.\frac{\partial Y_{E}}{\partial r}\right|_{r=a}-\left.\frac{\partial Y_{0}}{\partial r}\right|_{r=a}\right)=-\Delta Q_{0 S},
\end{gathered}
$$

where $\varepsilon_{h}^{\prime}$ and $\varepsilon_{s}^{\prime}$ are the dielectric constants of the system and the semiconductor, $Y_{0}$ is the equilibrium bend of the energy bands (at $V_{0}=0$ ) on the surface of the semiconductor particle, $\Delta Q_{0 S}$ is the change of the charge on the surface electron states at the boundary of the semiconductor particle, $k$ is the Boltzmann constant, $e$ is the absolute value of the electron charge, $T$ is the temperature, $\varepsilon_{0}$ is the dielectric constant, $r$ is the radius vector in the spherical coordinate system related to the center of the particle.

The changes of the dimensionless bending of the energy bands $Y_{0}$ and the distribution of the dimensionless potential in the environment surrounding particle $\tilde{\psi}_{0 d}\left(\tilde{\psi}_{0 d}=e \psi_{0 d} /(k T)\right)$ can be presented in the following form ${ }^{27,28}$ :

$$
\begin{gathered}
Y_{E}=Y_{0}+\sum_{n=1}^{\infty} Y_{E}^{(n)} P_{n}(\cos \theta) \\
\psi_{0 d}=\left(-E_{0} r+\frac{A_{0}^{(1)}}{r^{2}}\right) P_{1}(\cos \theta)+\sum_{n=2}^{\infty} \frac{A_{0}^{(n)}}{r^{(n+1)}} P_{n}(\cos \theta)
\end{gathered}
$$

In (3) and (4), $E_{0}$ is the mean macroscopic field that is formed by the constant voltage $V_{0}$ applied to the structure;
$P_{n}(\cos \theta)$ are the Legendre polynomials; $A_{0}^{(n)}$ and $Y_{E}^{(n)}$ are the unknown constants; $\theta$ is the polar angle of the spherical coordinate system.

The correctness of the expressions (3) and (4) is defined by the condition $L / a \ll 1$. It allows the decision of Poisson equation in spherical coordinate system to neglect surface currents and to reduce the problem to the plane in each point of the spherical surface. Thus, the solution is dependent on the polar angle $\theta$ of the spherical coordinate system with the center of the particle as the parameter.

Substituting the expansions (3) and (4) into the system (1) and (2) and equating terms with identical indices " $n$ " $(n=0,1, \ldots)$, one can come to the countless numbers of the equations systems to determine the constants $Y_{E}^{(n)}$. In particular, for finding the indicated unknown coefficients, corresponding dipole components of the potentials $Y_{E}(r, \theta)$ and $\psi_{0 d}(r, \theta)(n=1)$ can be obtained

$$
\begin{gathered}
Y_{E}^{(1)}=\left.\frac{e}{k T}\left(-E_{0} r+\frac{A_{0}^{(1)}}{r^{2}}\right)\right|_{r=a}, \\
\left.\varepsilon_{0} \varepsilon_{h}^{\prime}\left(-E_{0}-\frac{2 A_{0}^{(1)}}{r^{3}}\right)\right|_{r=a} \\
=\frac{3}{2} \int_{0}^{\pi}\left[\left.\frac{\partial\left(Y_{E}-Y_{0}\right)}{\partial r}\right|_{r=a}-\frac{e L \Delta Q_{0 S}}{\varepsilon_{0} \varepsilon_{s}^{\prime} k T}\right] \\
\quad \times P_{1}(\cos \theta) \cdot \sin \theta d \theta
\end{gathered}
$$

After expressing $A_{0}^{(1)}$ from (5) and having substituted the resulting expression in (6), we can obtain

$$
\begin{aligned}
-\frac{\varepsilon_{h}^{\prime}}{\varepsilon_{s}^{\prime}} & \left.\left(3 \tilde{E}_{0} L-\frac{2 Y_{E}^{(1)}}{\tilde{a}}\right)\right|_{r=a} \\
= & \frac{3}{2} \int_{0}^{\pi}\left[\left.\frac{\partial\left(Y_{E}-Y_{0}\right)}{\partial \tilde{r}}\right|_{r=a}-\frac{e L \Delta Q_{0 S}}{\varepsilon_{0} \varepsilon_{s}^{\prime} k T}\right] \\
& \times P_{1}(\cos \theta) \cdot \sin \theta d \theta,
\end{aligned}
$$

where $L=\left[\varepsilon_{0} \varepsilon_{s}^{\prime} k T /\left(e^{2} n_{0 V}^{-}\right)\right]^{1 / 2}, \tilde{E}_{0}=e E_{0} /(k T), \tilde{a}=a / L$, $\tilde{r}=r / L, n_{0 V}^{-}$is the bulk concentration of free electrons.

The dielectric loss factor considered structure can be found based on the results of calculation of the complex dielectric constant of the matrix system of spherical semiconductor particles in the dielectric. It is shown that the effective complex dielectric constant (which takes into account the polarization of the free charge) of the spherical semiconductor particles in a nonconducting medium may be determined by the expression ${ }^{29}$

$$
\varepsilon_{s}^{*}=\varepsilon_{s}^{\prime} / B^{*} .
$$

In the considered high-frequency range, the value $B^{*}=B_{h}$ is determined by the expression

$$
B_{h}=\frac{n_{I} L_{I}^{2}}{2 a}\left[\frac{\left(n_{0 S}^{+}-n_{0 V}^{+}\right)-\left(n_{0 S}^{-}-n_{0 V}^{-}\right)}{\partial Y_{0} /\left.\partial r\right|_{r=a}}\right]^{-1},
$$


where $L_{I}=\left[2 \varepsilon_{0} \varepsilon_{s}^{\prime} k T /\left(e^{2} n_{I}\right)\right]^{1 / 2}, n_{0 S}^{-}$and $n_{0 S}^{+}$are the surface concentrations of free electrons and holes; $n_{0 V}^{+}$is the bulk concentration of free holes; $n_{I}$ is the bulk concentration of free electrons in the intrinsic semiconductor.

Analyzing the method of calculation of this parameter, it is necessary to consider that the distributions of the concentration and potentials of the semiconductor particle when exposed to the dc electric field $E_{0}$ and the weak alternating electric field $E_{1} \exp (j \omega t)$ are represented by the general formula $\alpha(r, \theta, t)=\alpha_{0}(r, \theta)+\alpha_{1}(r, \theta) \exp (j \omega t)$.

The solution of the problem demands the account of both the electric dipole moments and higher electric moments of the polarized semiconductor sphere. The reason for this is that in determining the variable component of the electric dipole moment in the linearized equations of the continuity, the kinetics of the recharge localized states, the Poisson equation, the Laplace equation, the conditions of the potential continuity, the electric induction and currents at the boundary of the semiconductor particle only terms proportional to the multiplication of $\cos (\theta)$ on $\exp (j \omega t)$ are used, that $\alpha_{1}(r, \theta)=$ $\alpha_{1}(r) \cos (\theta) \exp (j \omega t)=\alpha_{1}(r) P_{1}(\cos (\theta)) \exp (j \omega t) \quad$ is assumed. Hence, it follows directly that in these equations, the angular dependence of the stationary potential and the concentration of charge carriers formed by the dc electric field in accordance with (3) and (4) will be lost. The expression for the variable component of the electric dipole moment and the value $B^{*}$ does not change in comparison with the case of absence of the dc electric field. ${ }^{29}$ For the range under consideration of the frequency, when recharging of surface electron states can be neglected, the expression is represented in the form (9).

The solution of the polarization problem with finding the multipole electric moments of order above dipole is the difficult problem. The following approximate algorithm is used more optimally for finding in the experiment of the correct expression for the field dependence of the high-frequency dielectric constant of the system of spherical semiconductor particles in the dielectric. This takes into account that in the dc electric field, the effective dielectric constant of the spherical semiconductor particles in the considered frequency range is caused by the capacitance of the space charge region which is located in one hemisphere of the particles. With increasing the external electric field impoverishing bend of the energy bands in it and its thickness are increased, its capacitance is decreased. The second space charge region located in the opposite hemisphere has a more large capacitance, since here the external electric field decreases impoverishing bend of the energy bands and its thickness.

In this approximation, Eq. (7) and expressions (8)-(9) can be used to analyze the data of the capacitance field effect in heterogeneous structures. In some cases, information about the physical properties of these materials can be obtained directly from the capacitance-voltage characteristic. In particular, for an inhomogeneous structure, when the semiconductor phase has unipolar conductivity $\left(n_{0 V}^{+} \approx 0\right)$, this task is simplified. It is necessary to take into account that at high external bias fields $\left(Y_{E} \gg Y_{0}\right)$ the value of the bending of the energy bands $Y_{E}$ and derivative $\partial Y_{E} / \partial r$ are big only in the depletion of the majority charge carriers that are part of the space charge region. This part is the hemisphere that is symmetric about an axis passing through its center and a point corresponding to the angular coordinate $\theta=0$. Taking this into account, the integral in (7) can be approximately calculated by the method of the trapezoids ${ }^{30}$

$$
-\frac{\varepsilon_{h}^{\prime}}{\varepsilon_{s}^{\prime}}\left(3 \tilde{E}_{0} L\right)=-\frac{3}{2} \frac{1}{2}\left(\left.\frac{\partial Y_{E}}{\partial \tilde{r}}\right|_{\theta=0}-\left.\frac{\partial Y_{0}}{\partial \tilde{r}}\right|_{\theta=0}\right)
$$

and to get

$$
\begin{array}{r}
\left.\frac{\partial Y_{E}}{\partial \tilde{r}}\right|_{\theta=0}=\left.\frac{\partial Y_{0}}{\partial \tilde{r}}\right|_{\theta=0}+\frac{4}{3} \frac{\varepsilon_{h}^{\prime}}{\varepsilon_{s}^{\prime}}\left(3 \tilde{E}_{0} L\right) \\
=\frac{1}{L}\left\{\left.\frac{\partial Y_{0}}{\partial r}\right|_{\theta=0}+4 \frac{\varepsilon_{h}^{\prime}}{\varepsilon_{s}^{\prime}}\left(\frac{e E_{0}}{k T}\right)\right\} .
\end{array}
$$

It is accepted that at the not very large intensities of the applied electric field $\partial\left(Y_{E}-Y_{0}\right) /\left.\partial \tilde{r}\right|_{\theta=\pi / 2} \approx 0$. From the condition $L / a \ll 1$ it follows that in (7) $3 \tilde{E}_{0} L \gg 2 Y_{E}^{(1)} / \tilde{a}=2 Y_{E}^{(1)} \cdot(L / a)$.

Replacing in (9) $\partial Y_{0} /\left.\partial r\right|_{r=a}$ on the expression (11) for $\partial Y_{E} /\left.\partial r\right|_{\substack{r=a \\ \theta=0}}$ and considering that $n_{0 S}^{+} \sim n_{0 V}^{+} \approx 0$,

$$
n_{0 S}^{-}-n_{0 V}^{-}=n_{0 V}^{-}\left[\exp \left(-\left|Y_{E}\right|_{\substack{r=a \\ \theta=0}}\right)-1\right] \cong n_{0 V}^{-}
$$

and

$$
\partial Y_{0} /\left.\partial r\right|_{r=a}=\frac{1}{L}\left[-Y_{0}(a)-1\right]^{1 / 2}
$$

can be obtained

$$
\begin{aligned}
B_{h} & =\frac{n_{I} L_{I}^{2}}{2 a}\left[\frac{n_{0 V}^{-}}{\partial Y_{0} /\left.\partial r\right|_{r=a}}\right]^{-1} \\
& =\frac{L^{2}}{2 a}\left\{\frac{1}{L}\left[-Y_{0}(a)-1\right]^{1 / 2}+4 \frac{\varepsilon_{h}^{\prime}}{\varepsilon_{s}^{\prime}}\left(\frac{e E_{0}}{k T}\right)\right\} .
\end{aligned}
$$

\section{Capacitance-Voltage Characteristics}

The metal oxide ceramics and the composite materials based on semiconductors have the dispersed structure. The specific parameters and the characteristics are more appropriate to describe the properties of the metal oxide ceramics and the composite materials. In particular, the relative dielectric constant $\varepsilon_{h}^{\prime}$ of such materials is correctly used to describe the capacitance properties in the considered frequency range. The dependence of the dielectric constant from the applied dc electric field $\varepsilon_{h}^{\prime}\left(E_{0}\right)$ is considered as the capacitance-voltage characteristic of these materials. It is accepted later in this paper. 


\subsection{Matrix system}

The method based on using the model of spherical semiconductor particles in the dielectric matrix is the most correct one to solve the problem of determining complex dielectric constant of matrix systems. The perspective of this model is substantiated by usually existing chaotic orientation of particles of arbitrary shape in many known materials. It is experimentally confirmed by the isotropy of their electrical properties. The essence of this approach consists in calculating electric dipole moment of the separate particle $d_{e}$ and determining on the basis of this dipole moment of the complex dielectric constant of the matrix system by the methods of the theory of the two-component dielectrics. The integral method (or the method of self-consistent field) is recognized as the most successful approximate method of calculation of the equivalent dielectric constant of two-component matrix systems. ${ }^{28,31}$ Within this method, an increment of the dielectric constant of the system $d \varepsilon^{*}$ is considered upon introduction in it of a small bulk fraction of dispersed particles $d p_{V}$. The differential equation is made on that basis. The result of the integration of this equation with the initial condition $\varepsilon^{*}\left(p_{V}=0\right)=\varepsilon_{d}^{\prime} \quad\left(\varepsilon_{d}^{\prime}\right.$ is the relative dielectric constant of the dielectric of the matrix) leads to the Bruggeman-Hanai equation. ${ }^{28,31-33}$ In the case considered of the high-frequency dielectric constant, this formula looks like

$$
\left(\varepsilon_{h}^{\prime}-\varepsilon_{s}^{\prime} / B_{h}\right) /\left(\varepsilon_{d}^{\prime}-\varepsilon_{s}^{\prime} / B_{h}\right) \cdot\left(\varepsilon_{d}^{\prime} / \varepsilon_{h}^{\prime}\right)^{1 / 3}=1-p_{V},
$$

where $p_{V}$ is the bulk fraction of the semiconductor particles in the heterogeneous system.

The field dependence of the high-frequency dielectric constant $\varepsilon_{h}^{\prime}\left(E_{0}\right)$ is shown in Fig. 1. The typical parameter values of the zinc oxide varistor ceramics $\varepsilon_{h}^{\prime}=10, \varepsilon_{s}^{\prime}=10$, $Y_{0}(a)=-30, n_{0 V}^{-}=10^{17} \mathrm{~cm}^{-3}$ have been accepted in the calculations.

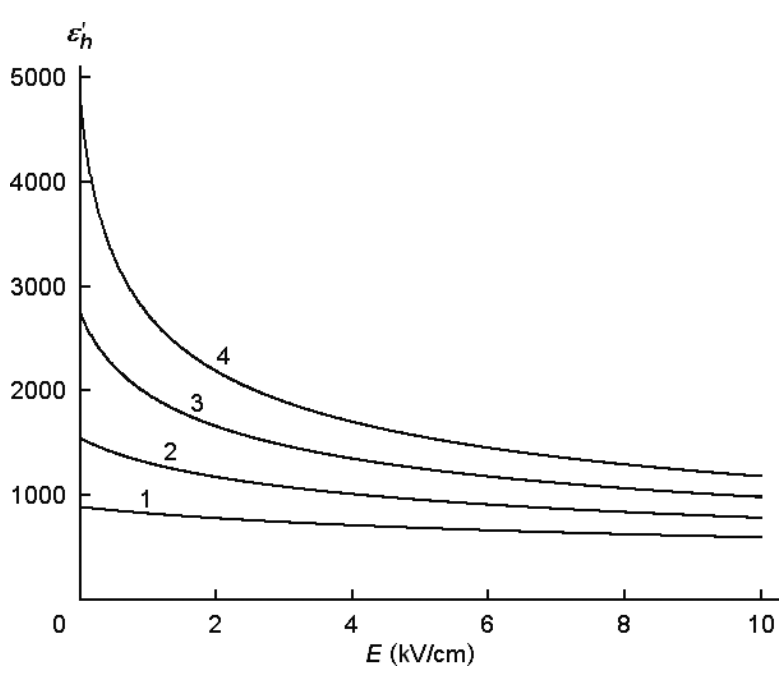

Fig. 1. The field dependence of the relative dielectric constant of the matrix system for the bulk fractions $p_{V}: 1-0.8 ; 2-0.85 ; 3-0.9$; $4-0.95$.

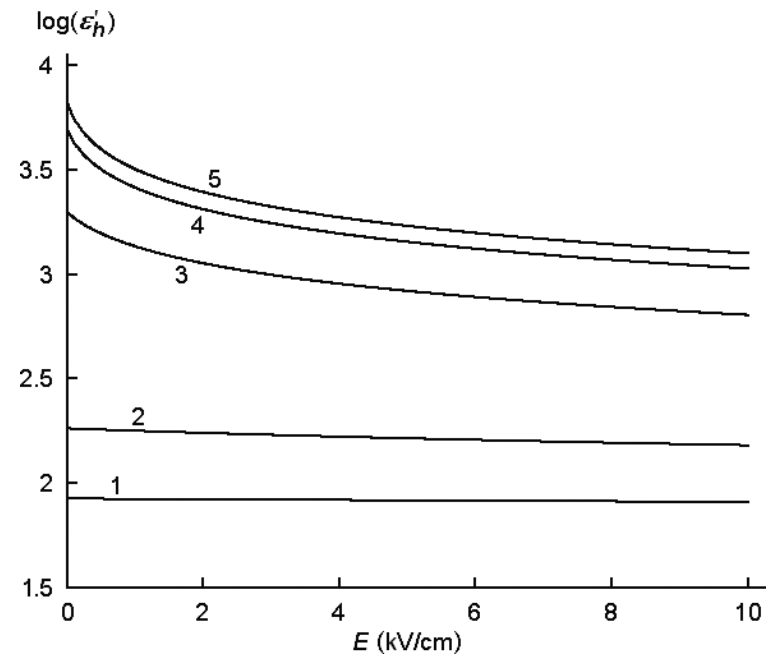

Fig. 2. The field dependence of the relative dielectric constant of the statistical mixture for the bulk fractions $p_{V}: 1-0.3 ; 2-0.33$; $3-0.5 ; 4-0.75 ; 5-0.9$.

Significant dependence of the absolute values $\varepsilon_{h}^{\prime}$ is observed at the change of the concentration of the particles of the semiconductor $p_{V}$. The dielectric constant nonlinearly decreases with increasing the applied electric field. The value of this change $\varepsilon_{h}^{\prime}$ increases with increasing $p_{V}$.

\subsection{Statistical mixture}

The expression for the complex dielectric constant of the statistical mixture of semiconductor and insulator $\varepsilon^{*}$ is obtained by determining the polarization vector of the unit bulk of this mixture. This vector is found by summing the polarization vectors of all semiconductor and dielectric particles. $^{28,33}$ As a result, the Böttcher-Hsu equation for the spherical particles is obtained. ${ }^{32-34}$ This equation for the highfrequency dielectric constant can be represented in the form

$$
\begin{aligned}
& \left(\varepsilon_{s}^{\prime} / B_{h}-\varepsilon_{h}^{\prime}\right) /\left(\varepsilon_{s}^{\prime} / B_{h}+2 \varepsilon_{h}^{\prime}\right) \cdot p_{V} \\
& \quad+\left(\varepsilon_{d}^{\prime}-\varepsilon_{h}^{\prime}\right) /\left(\varepsilon_{d}^{\prime}+2 \varepsilon_{h}^{\prime}\right) \cdot\left(1-p_{V}\right)=0 .
\end{aligned}
$$

The corresponding field dependences of the highfrequency dielectric constant $\varepsilon_{h}^{\prime}\left(E_{0}\right)$ are shown in Fig. 2.

As it is evident, the closer the value of the bulk fraction of semiconductor particles $p_{V}$ to the threshold of its value $p_{C}=1 / 3,33,35$ the larger the absolute values $\varepsilon_{h}^{\prime}$ and the strong dependence $\varepsilon_{h}^{\prime}$ from the magnitude of the applied electric field $E_{0}$.

\section{Features of the Application of the Models for Determining of the Bulk Donor Concentration}

\subsection{Adaptation of the models for analysis of experimental data}

By determining the concentration of bulk donors in the semiconductor component under the consideration of the inhomogeneous systems from its capacitance-voltage characteristics, 
expression (12) can be transformed into the following form:

$$
\begin{aligned}
B_{h}\left(E_{0}\right) & =B_{h}(0)+\frac{L^{2}}{2 a} \frac{\varepsilon_{h}^{\prime}\left(E_{0}\right)}{\varepsilon_{s}^{\prime}} \frac{4 e E_{0}}{k T} \\
& =B_{h}(0)+\frac{2 \varepsilon_{0} \varepsilon_{h}^{\prime}\left(E_{0}\right) \cdot E_{0}}{e n_{0 V}^{-} a}
\end{aligned}
$$

where the quantities $\varepsilon_{h}^{\prime}\left(E_{0}\right), B_{h}\left(E_{0}\right), B_{h}(0)$ are the values of the corresponding parameters $\varepsilon_{h}^{\prime}$ and $B_{h}$ in the presence of dc electric field $(E \neq 0)$ and in its absence $(E=0)$ determined from the experimental capacitance-voltage characteristics.

The value $B_{h}$ for the matrix system can be determined based on expressions (13)

$$
B_{h}=\frac{\varepsilon_{s}^{\prime}}{\varepsilon_{d}^{\prime}} \frac{1-\left(1-p_{V}\right) \cdot\left(\varepsilon_{h}^{\prime} / \varepsilon_{d}^{\prime}\right)^{1 / 3}}{\left(\varepsilon_{h}^{\prime} / \varepsilon_{d}^{\prime}\right)-\left(1-p_{V}\right) \cdot\left(\varepsilon_{h}^{\prime} / \varepsilon_{d}^{\prime}\right)^{1 / 3}}
$$

and the value $B_{h}$ for the statistical mixture can be determined based on (14)

$$
B_{h}=\frac{\varepsilon_{s}^{\prime}}{\varepsilon_{h}^{\prime}} \frac{1-A}{1+2 A},
$$

where $A=-\frac{1-p_{V}}{p_{V}} \cdot \frac{\varepsilon_{d}^{\prime}-\varepsilon^{\prime}}{\varepsilon^{\prime}+2 \varepsilon^{\prime}}$.

The following relation for determining the concentration of the bulk donors in semiconductor particles $N_{d}=n_{0 V}^{-}$can be obtained based on the expression (15)

$$
n_{0 V}^{-}=\frac{2 \varepsilon_{0}}{e a_{s}} \cdot \Delta\left[\varepsilon_{h}^{\prime}\left(E_{0}\right) \cdot E_{0}\right] / \Delta B_{h}\left(E_{0}\right),
$$

where $\Delta B_{h}\left(E_{0}\right) / \Delta\left[\varepsilon_{h}^{\prime}\left(E_{0}\right) \cdot E_{0}\right]$ is tangent of the inclination angle of the experimental dependence of the parameter $B_{h}\left(E_{0}\right)$ from the multiplication $\varepsilon_{h}^{\prime}\left(E_{0}\right) \cdot E_{0}$.

\subsection{Comparison of the experimental results of applying different models}

\subsubsection{Zinc oxide varistor ceramics}

In the work, zinc oxide commercial high-voltage ceramics for varistors $\mathrm{SN} 2-2$ of composition $\mathrm{ZnO}-\mathrm{Bi}_{2} \mathrm{O}_{3}-\mathrm{CoO}-\mathrm{Sb}_{2} \mathrm{O}_{3}-$ $\mathrm{SnO}_{2}-\mathrm{MnO}-\mathrm{B}_{2} \mathrm{O}_{3}$ were used.

These ceramics have typical microstructure as semiconductor zinc oxide grains separated by a dielectric interlayer that has been described in many research. ${ }^{17,18,36}$ The evaluation of the bulk fraction of the dispersed phase of the $\mathrm{ZnO}$ was obtained from the carried out chemical composition of the studied ceramics and was the value of order 0.8 . The sizes of grains were measured with the use of optic microscopy. These sizes had the average value of $10 \mu \mathrm{m}$. The samples were in the form of flat round tablets of diameter $2.6 \mathrm{~cm}$ and thickness $\sim 0.13 \mathrm{~cm}$. Threshold voltage (voltage across the varistor measured at a given reference current $1 \mathrm{~mA} \mathrm{dc}$ ) was $\sim 137 \mathrm{~V}$.

The range of radio frequencies from $10 \mathrm{~Hz}$ to $10^{4} \mathrm{~Hz}$ is used in the study of capacitance-voltage characteristics of commercial zinc oxide ceramics. ${ }^{6,7}$ In this range, the above formulated condition of high-frequency capacitance-voltage characteristics is executed. The known fields of the dispersion of the dielectric constant for zinc oxide are connected with recharging localized electronic states and the MaxwellWagner relaxation of free charge carriers. The first of these fields is observed in the infra-low frequencies, and the second is observed in the $\mathrm{GHz}$ range. ${ }^{29}$

For investigation of capacitance-voltage characteristics, the measurement scheme described in work $^{7,9}$ was used which allows one to measure capacitance in a wide range of direct shifting voltage $(0-700 \mathrm{~V})$ and frequencies of alternating current $(20 \mathrm{~Hz}-50 \mathrm{kHz})$.

The sample was connected to dc power source through the inductance. Its value was chosen to be sufficiently large in order to avoid the influence of direct current circuit on the results of measurements under the alternating current. An alternating current bridge circuit was connected to the samples via a blocking capacitor, which did not let the direct current component to the bridge. Monitoring of the direct voltage on the sample and the current through it was done with a voltmeter and a milliammeter, respectively.

It is known that the varistor ceramics by the microstructure represent the highly concentrated heterogeneous structures of the matrix type with large bulk fractions of zinc oxide grains $p_{V} \cdot{ }^{6,17,18}$ For such highly concentrated matrix systems in which the value $p_{V} \rightarrow 1$ can be accepted

$$
B_{h}=\varepsilon_{s}^{\prime} / \varepsilon_{h}^{\prime}
$$

and the expression (18) can be simplified

$$
n_{0 V}^{-}=\frac{2 \varepsilon_{0}}{e a \varepsilon_{s}^{\prime}} \cdot \Delta\left[\varepsilon_{h}^{\prime}\left(E_{0}\right) \cdot E_{0}\right] / \Delta \frac{1}{\varepsilon_{h}^{\prime}\left(E_{0}\right)},
$$

where $\Delta\left[\varepsilon_{h}^{\prime}\left(E_{0}\right) \cdot E_{0}\right]$ and $\Delta\left[1 / \varepsilon_{h}^{\prime}\left(E_{0}\right)\right]$ are the increments of the corresponding quantities when the applied dc electric field $E_{0}$ to the structure changes.

The experimental capacitance-voltage characteristics of the considered varistor ceramics are given in Fig. 3. The dependency (1) is shown in the coordinates used to estimate $N_{d}$ by the one-dimensional model ${ }^{16}$

$$
\frac{1}{\varepsilon_{h}^{\prime}\left(E_{0}\right)}=\frac{1}{\varepsilon_{d}^{\prime}} \frac{\Delta d}{d}+\left(\frac{2 \varepsilon_{0} E_{0}}{e \varepsilon_{s}^{\prime} N_{d} d}\right)^{1 / 2}
$$

where $\Delta d$ is the thickness of the interlayer of the matrix dielectric; $d$ is the thickness of the structure.

The results of the evaluation of the concentration of the bulk donors in the studied ceramics are shown in Table 1. The expression has been used in the calculation $N_{d}$ based on the one-dimensional model

$$
N_{d}=2 \varepsilon_{0} /\left(e \varepsilon_{s}^{\prime} d \kappa_{1 E}^{2}\right),
$$

where $\kappa_{1 E}$ is tangent of the inclination angle of the experimental dependence $1 / \varepsilon^{\prime}\left(E_{0}\right)$ on $E_{0}^{1 / 2}$. 


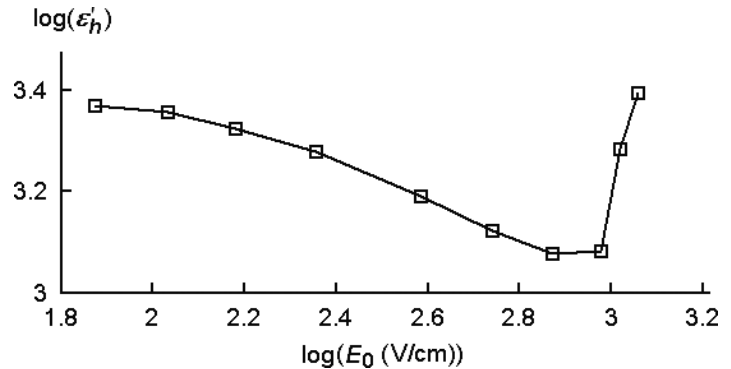

(a)

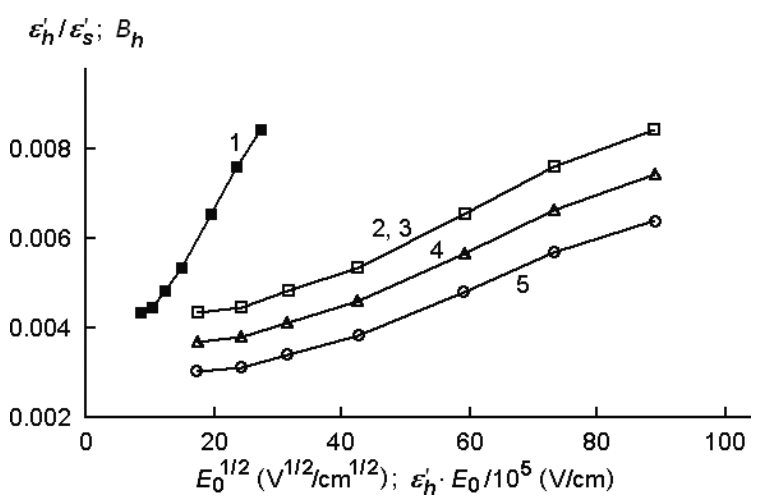

(b)

Fig. 3. The typical experimental field dependence of the high-frequency dielectric constant of the varistor ceramics sample (a) and its representation in the coordinates: $1-\left(\varepsilon_{s}^{\prime} / \varepsilon_{h}^{\prime}, E_{0}^{1 / 2}\right) ; 2-\left(\varepsilon_{s}^{\prime} / \varepsilon_{h}^{\prime}, \varepsilon_{h}^{\prime} \cdot E_{0}\right)$ and $3,4,5-\left(B_{h}, E_{0}^{1 / 2}\right)$ for $p_{V}$ equal $1,0.975$ and 0.95 , respectively (b).

The values $N_{d}$ are shown in Table 1 . These data are obtained by using well-known in the literature of the onedimensional models as well as analyzed by three-dimensional model of the matrix system in this paper. These data demonstrate the one and the same order of magnitude values $N_{d}$. The latest model gives the somewhat smaller value $N_{d}$. This can be considered in favor of its more correctness. However, providing of this correctness is connected with precise knowledge of the magnitude of the bulk fraction of the semiconductor particles in the system. Reducing the bulk fraction also increases the magnitude estimation of the defined parameter.

\subsubsection{Influence of the bulk fraction of the semiconductor component on the measurement results}

In practical use of the method of the capacitance-voltage characteristics, the exact value of such parameter as the value of the bulk fraction of semiconductor particles in the heterogeneous structure is not known as a rule. Thus, the use of calculation formulas (15)-(17) is problematic. In this connection, it seems appropriate to analyze the level of errors to which the use of the relations (20) leads. It does not take into account the influence of the magnitude of the bulk fraction of the semiconductor components on the measurement results instead of (18).

Table 1. The estimates of the concentration of the bulk donors in varistor ceramics based on different models.

\begin{tabular}{lccc}
\hline \multicolumn{1}{c}{ Model } & & $N_{d} \cdot 10^{-17}, \mathrm{~cm}^{-3}$ & Note \\
\hline $\begin{array}{l}\text { One-dimensional } \\
\quad \text { (layered system) }\end{array}$ & Eq. (21) & 1.9 & \\
$\begin{array}{l}\text { Three-dimensional } \\
\quad \text { (system of the }\end{array}$ & Eq. (20) & 1.7 & \\
$\quad$ spherical particles & Eqs. (16) and (18) & 1.7 & $p_{V}=1$ \\
in the dielectric & & 1.8 & $p_{V}=0.975$ \\
$\quad$ matrix) & & 2.0 & $p_{V}=0.95$ \\
\hline
\end{tabular}

For this, the value of the donor concentration $N_{d}$ $\left(N_{d}=10^{17} \mathrm{~cm}^{-3}\right)$ was set. The calculation of the theoretical capacitance-voltage characteristic in the form $\varepsilon_{h}^{\prime}\left(E_{0}\right)$ based on (13) taking into account (12) for different values of the bulk fraction of the semiconductor particles $p_{V}$ was produced. The obtained dependence was used to estimate the donor concentration $N_{d}^{*}$ according to (20) and the relative magnitude of the change of the high-frequency dielectric constant of the structure $\Delta \varepsilon_{h}\left(E_{0 \max }\right)=\left(\varepsilon_{h}^{\prime}(0)-\varepsilon_{h}^{\prime}\left(E_{0 \max }\right)\right) / \varepsilon_{h}^{\prime}(0)$ at increased dc voltage from 0 till $E_{0 \max }=10^{3} \mathrm{~V} / \mathrm{cm}$ (Table 2). The analogous parameters for the statistical mixture of semiconductor and dielectric particles are given.

As can be seen from Table 2, the sensitivity of the relative dielectric constant to the applied dc electric field is dependent on the bulk fraction of the semiconductor particles in the heterogeneous structure. The substantial (exceeding a few percent) decrease of the dielectric constant with increasing by applied external electric field used in the experiment of the range begins with the value $p_{V}$ from $\sim 0.8$ for the matrix systems and from $\sim 0.33$ for the statistical mixtures.

In the table, it is accepted that the minimum change of the dielectric constant, which can be fixed, corresponds to the

Table 2. The dependence of the estimates of the donor concentration $N_{d}^{*}$ in the semiconductor particles on the approximate formula (20) from their bulk fraction $p_{V}$ in the heterogeneous structures.

\begin{tabular}{lccccc}
\hline & \multicolumn{2}{c}{ Matrix system } & & \multicolumn{2}{c}{ Statistical mixture } \\
\cline { 2 - 3 } \cline { 5 - 6 }$p_{V}$ & $\Delta \varepsilon_{h}\left(E_{0 \max }\right)$ & $N_{d}^{*} \cdot 10^{-16}, \mathrm{~cm}^{-3}$ & & $\Delta \varepsilon_{h}\left(E_{0 \max }\right)$ & $N_{d}^{*} \cdot 10^{-16}, \mathrm{~cm}^{-3}$ \\
\hline 0.3 & $<0.01$ & - & & $<0.01$ & - \\
0.33 & $<0.01$ & - & & 0.03 & 3.7 \\
0.4 & $<0.01$ & - & & 0.27 & 1.1 \\
0.5 & $<0.01$ & - & & 0.42 & 2.5 \\
0.6 & $<0.01$ & - & & 0.50 & 4.0 \\
0.7 & $<0.01$ & - & & 0.55 & 5.5 \\
0.8 & 0.05 & 3.9 & & 0.59 & 7.0 \\
0.9 & 0.30 & 5.3 & & 0.62 & 8.5 \\
0.95 & 0.49 & 7.2 & & 0.63 & 9.3 \\
0.975 & 0.57 & 8.5 & & 0.63 & 9.6 \\
\hline
\end{tabular}


relative change on $1 \%$. According to the results presented here, the model of the statistical mixture of the semiconductor and dielectric particles predicts greater sensitivity to the applied electric field.

It should also be noted that one of the essential conditions of the observation under consideration here of the effect of the capacitance reducing in the dc electric field is the presence in the particles of the semiconductor of the depleted bend of the energy bands, as well as for one-dimensional models of this capacitance effect.

\section{Conclusions}

The ratio that allows to estimate the value of the concentration of the bulk of donors in the semiconductor component of heterogeneous systems in accordance with the ideas of their structure as a disperse two-component medium is given.

The sensitivity of the relative dielectric constant of such systems to an external direct electric field is dependent on the bulk fraction of semiconductor particles in them. For matrix systems within the Bruggeman-Hanai model, the influence of the bulk fraction is significant beginning with the values from $\sim 0.8$, and for statistical mixtures based on the Böttcher-Hsu model, the influence of the bulk fraction is significant beginning with the values from $\sim 0.33$.

The use of the calculated ratios to evaluate the donor concentration in the $\mathrm{ZnO}$ grains of the zinc oxide varistor ceramics leads to values that are consistent with those of other methods.

\section{References}

${ }^{1}$ L. Y. Leu and S. R. Forrest, The determination of heterojunction energy band discontinuities in the presence of interface states using capacitance-voltage techniques, J. Appl. Phys. 64, 5030 (1988).

${ }^{2}$ V. Y. Aleshkin, E. V. Demidov, B. N. Zvonkov, A. V. Murel and Y. A. Romanov, Study of quantum wells by the C-V method, Sov. Phys. Semicond. 25, 631 (1991).

${ }^{3}$ S. Karataş, F. Yakuphanoglu and F. M. Amanullah, Capacitancevoltage and conductance-voltage characteristics of $\mathrm{Ag} / \mathrm{n}-\mathrm{CdO} / \mathrm{p}-$ Si MIS structure prepared by sol-gel method, J. Phys. Chem. Solids 73, 46 (2012).

${ }^{4}$ A. V. Voitsekhovskii, S. N. Nesmelov and S. M. Dzyadukh, Capacitance-voltage characteristics of metal-insulator-semiconductor structures based on graded-gap $\mathrm{HgCdTe}$ with various insulators, Thin Solid Films 522, 261 (2012).

${ }^{5}$ M. Moertelmaier, H. P. Huber, C. Rankl and F. Kienberger, Continuous capacitance-voltage spectroscopy mapping for scanning microwave microscopy, Ultramicroscopy 136, 67 (2014).

${ }^{6}$ V. B. Kvaskov, Semiconductor Devices with Bipolar Conductivity (Energoatomizdat, Moscow, 1988) (in Russian).

${ }^{7} \mathrm{G}$. E. Pike, Electronic properties of $\mathrm{ZnO}$ varistors: A new model, Grain boundaries in semiconductors, Proceedings of the Material Research Society Annual Meeting, eds. H. J. Leamy, G. E. Pike and C. H. Seager (Elsevier Science Publishing Company, Boston, MA, 1982), pp. 369-379.
${ }^{8} \mathrm{R}$. Einzinger, Grain junction properties of $\mathrm{ZnO}$ varistors, Appl. Surf. Sci. 3, 390 (1979).

${ }^{9}$ A. S. Tonkoshkur, I. V. Gomilko and A. Yu. Lyashkov, Percolation effects in the capacitive properties of metal-oxide varistors in the range of high voltage, J. Adv. Dielect. 4, 1450013 (2014).

${ }^{10}$ A. S. Tonkoshkur and A. V. Ivanchenko, The effect of negative capacitance in varistor structure on the basis of its models with voltage drop on the intergranular interlayer, Multidiscipl. Model. Mater. Struct. 11, 598 (2015).

${ }^{11}$ P. R. Bueno, M. R. de Cassia-Santos, E. R. Leite, E. Longo, J. Bisquert, G. Garcia-Belmonte and F. Fabregat-Santiago, Nature of the Schottky-type barrier of highly dense $\mathrm{SnO}_{2}$ systems displaying nonohmic behavior, J. Appl. Phys. 88, 6545 (2000).

${ }^{12}$ M. H. Wang, Q. H. Tang and C. Yao, Electrical properties and AC degradation characteristics of low voltage $\mathrm{ZnO}$ varistors doped with $\mathrm{Nd}_{2} \mathrm{O}_{3}$, Ceram. Int. 36, 1095 (2010).

${ }^{13}$ J. He, W. Long, J. Hu and J. Liu, Nickel oxide doping effects on electrical characteristics and microstructural phases of $\mathrm{ZnO}$ varistors with low residual voltage ratio, J. Ceram. Soc. Jpn. 119, 43 (2011).

${ }^{14} \mathrm{C}$. W. Nahm, Major effects on varistor properties of $\mathrm{ZnO}-\mathrm{V}_{2} \mathrm{O}_{5}-$ $\mathrm{MnO}_{2}-\mathrm{Nb}_{2} \mathrm{O}_{5}-\mathrm{Er}_{2} \mathrm{O}_{3}$ ceramics with sintering changes, Ceram. Int. 38, 2593 (2012).

${ }^{15}$ Y. Tu, Z. Zheng, X. Li, Q. Wang and M. Luo, Grain-boundary and thermally stimulated current characteristics of $\mathrm{Y}_{2} \mathrm{O}_{3}$-doped $\mathrm{ZnO}$ varistor, J. Am. Ceram. Soc. 96, 3518 (2013).

${ }^{16}$ A. S. Tonkoshkur and I. M. Chernenko, The capacitance-voltage characteristic of the symmetric structure of the semiconductordielectric, Dielektriki i Poluprovodniki. Respublikanskiy Meduzhvedomstvennyiy Nauchno-Tehnicheskiy Sbornik. Kiev: Vischa shkola 23, 63 (1983) (in Russian).

${ }^{17}$ T. K. Gupta, Application of zinc oxide varistors, J. Am. Ceram. Soc. 73, 1817 (1990).

${ }^{18}$ D. R. Clarke, Varistor ceramics, J. Am. Ceram. Soc. 82, 485 (1999).

${ }^{19}$ K. Mukae, K. Tsuda and I. Nagasawa, Capacitance-vs-voltage characteristics of $\mathrm{ZnO}$ varistors, J. Appl. Phys. 50, 4475 (1979).

${ }^{20}$ J. J. Lee, J. K. O'brien and M. S. Cooper, Second-breakdown characteristics of metal-oxide varistors, J. Appl. Phys. 48, 1252 (1977).

${ }^{21}$ H. R. Philipp and L. M. Levinson, Optical method for determining the grain resistivity in $\mathrm{ZnO}$-based ceramic varistors, J. Appl. Phys. 47, 1112 (1976).

${ }^{22}$ E. F. Afonko and A. S. Tonkoshkur, Influence of charging of local centers on the electrical-properties of inhomogeneous CdS photoconducting structures, Sov. Phys.-Semicond.-(USSR) 16, 1224 (1982).

${ }^{23}$ A. M. Hashimov, S. M. Hasanli, R. N. Mehdizadeh, S. M. Azizova and K. B. Bayramov, Nonlinear resistor based on a polymer-ceramic composition, Tech. Phys. 52, 1086 (2007).

${ }^{24}$ C. C. Lin, W. S. Lee, C. C. Sun and W. H. Whu, A varistorpolymer composite with nonlinear electrical-thermal switching properties, Ceram. Int. 34, 131 (2008).

${ }^{25}$ A. Y. Lyashkov and A. S. Tonkoshkur, Varistor composites with a positive temperature coefficient of resistance, Tech. Phys. 56, 427 (2011).

${ }^{26}$ S. Afroze, H. Kabir, M. M. Rahman, M. A. Kabir, F. Ahmed, M. A. Hossain and M. A. Gafur, Elastic and electrical properties of graphite and talc filler reinforced polypropylene (PP) composites, Int. J. Basic Appl. Sci. IJBAS-IJENS 12, 13 (2012). 
${ }^{27}$ A. S. Tonkoshkur, Volt-farad characteristic of inhomogeneous structure, Ukr. Fiz. Zh. 26, 1740 (1981) (in Russian).

${ }^{28} \mathrm{~S}$. S. Dukhin and V. N. Shilov, Dielectric Phenomena and the Double Layer in Disperse Systems and Polyelectrolytes (John Wiley \& Sons, New York, Toronto, 1974).

${ }^{29}$ A. S. Tonkoshkur, Effect of charge exchange of local centers on dielectric phenomena in disperse semiconductor structures, $U \mathrm{kr}$. Fiz. Zh. 23, 2030 (1978) (in Russian).

${ }^{30}$ T. E. Shoup, A Practical Guide to Computer Methods for Engineers (Prentice-Hall, Englewood Cliffs, New Jersey, 1979).

${ }^{31}$ T. Hanai, A remark on the "Theory of the dielectric dispersion due to the interfacial polarisation", Colloid Polym. Sci. 175, 61 (1961).
${ }^{32} \mathrm{G}$. Bànhegyi, Comparison of electrical mixture rules for composites, Colloid Polym. Sci. 264, 1030 (1986).

${ }^{33} \mathrm{G}$. Bànhegyi, Numerical analysis of complex dielectric mixture formulae, Colloid Polym. Sci. 266, 11 (1988).

${ }^{34}$ W. Y. Hsu, T. D. Gierke and C. J. Molnar, Morphological effects on the physical properties of polymer composites, Macromolecules 16, 1945 (1983).

${ }^{35}$ S.-G. Shin and I.-K. Kwon, Effect of temperature on the dielectric properties of carbon black-filled polyethylene matrix composites below the percolation threshold, Electron. Mater. Lett. 7, 249 (2011).

${ }^{36} \mathrm{~A}$. I. Ivon, Parameters of $\mathrm{ZnO}$ grains in $\mathrm{ZnO}$-based ceramics, Ing. Mater. 36, 1074 (2000). 